\title{
Characterization of liquid-liquid extraction fractions from lignocellulosic biomass by high performance liquid chromatography hyphenated to tandem high-resolution mass spectrometry (HPLC/MS $\left.{ }^{n}\right)$
}

Carole Reymond ${ }^{a}$, Alexis Dubuis ${ }^{a}$, Agnès Le Masle ${ }^{a,},{ }^{*}, C$ Cyril Colas ${ }^{b, c}$, Ludovic Chahen ${ }^{a}$, Emilie Destandau $^{\mathrm{b}}$, Nadège Charon ${ }^{\mathrm{a}}$

a IFP Energies nouvelles, Rond-point de l'échangeur de Solaize, BP 3, 69360 Solaize, France

${ }^{b}$ Institut de Chimie Organique et Analytique, Université d'Orléans, CNRS UMR 7311, Rue de Chartres, 45067 Orléans, France

'Centre de Biophysique Moléculaire, CNRS UPR 4301, Université d'Orléans, rue Charles Sadron, 45071 Orléans, France

*Corresponding author. E-mail address: agnes.le-masle@ifpen.fr (A. Le Masle)

\section{Supplementary material}

Table S1: Experimental log K obtained by GC-FID for the system MTBE/acetate buffer solution at $\mathrm{pH}=$ 2.8 and theoretical values log $\mathrm{P}$ related to chemical structures.

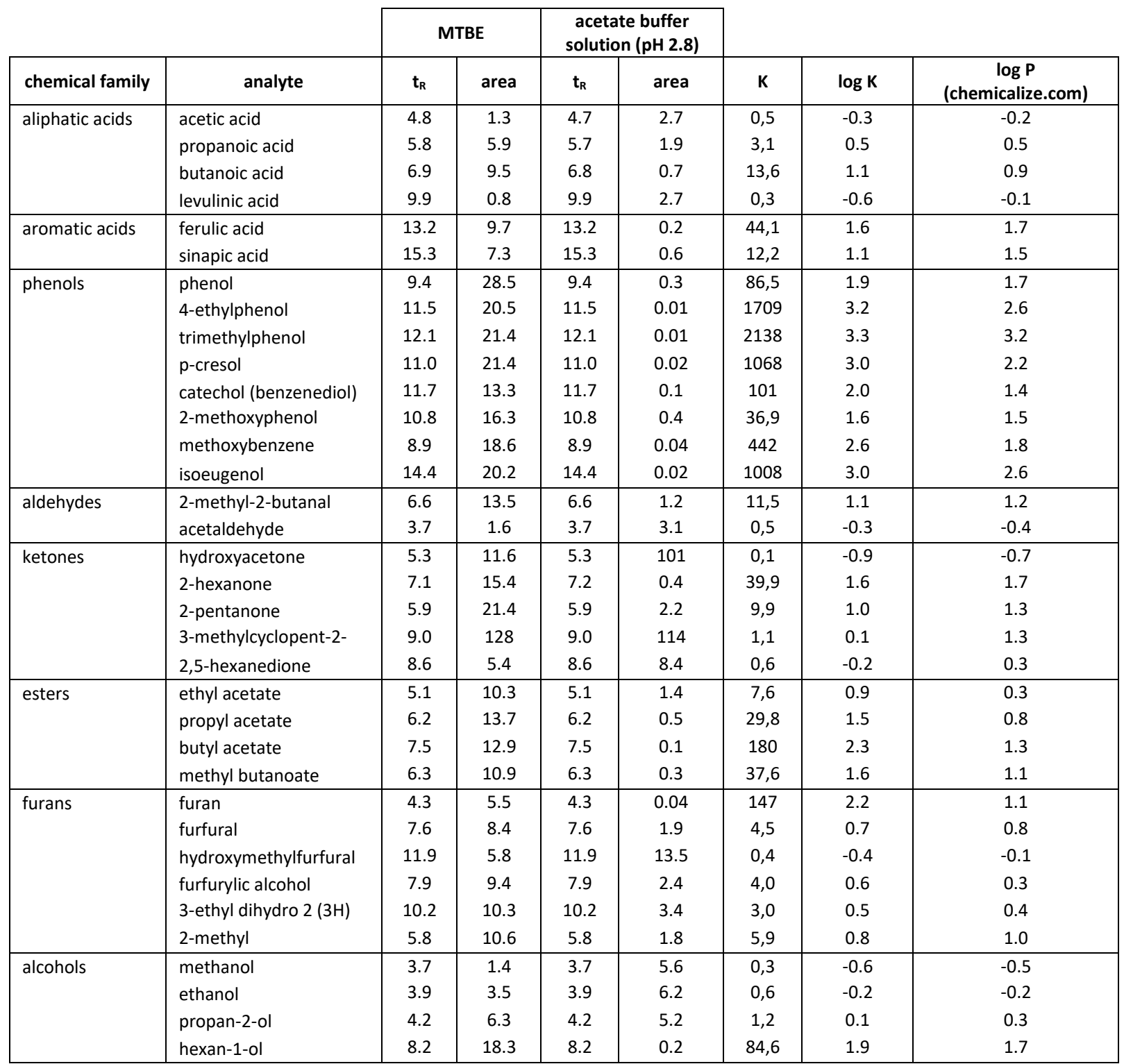


Figure S1: Partition coefficients of 35 model molecules representative of biomass chemical families in MTBE/water solvent system at $\mathrm{pH} 2.8$.

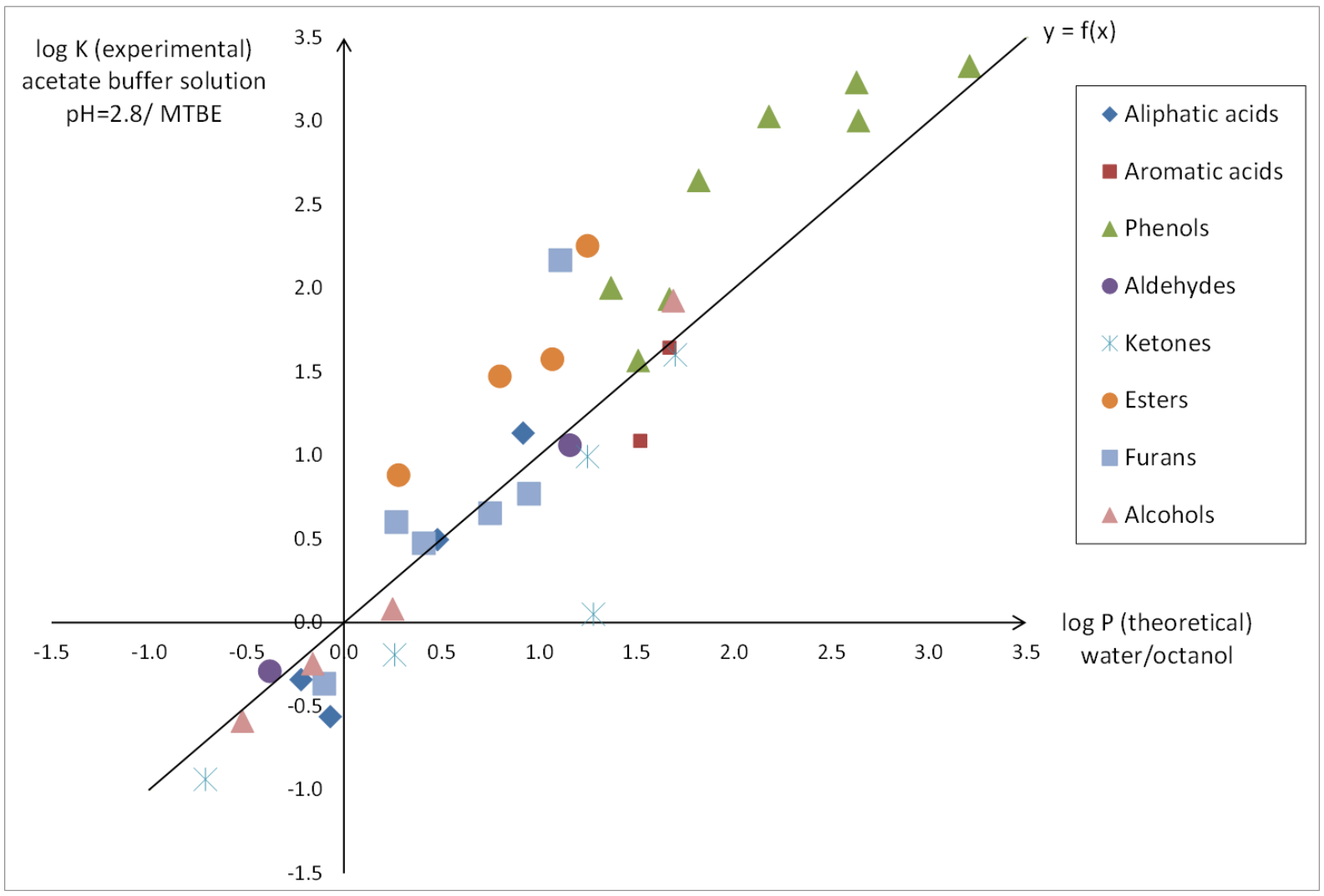

Figure S2: Mass distribution in the four fractions and the lost masses. Histograms correspond to the average mass percentage of each chemical family obtained after 3 repetitions on model molecules.

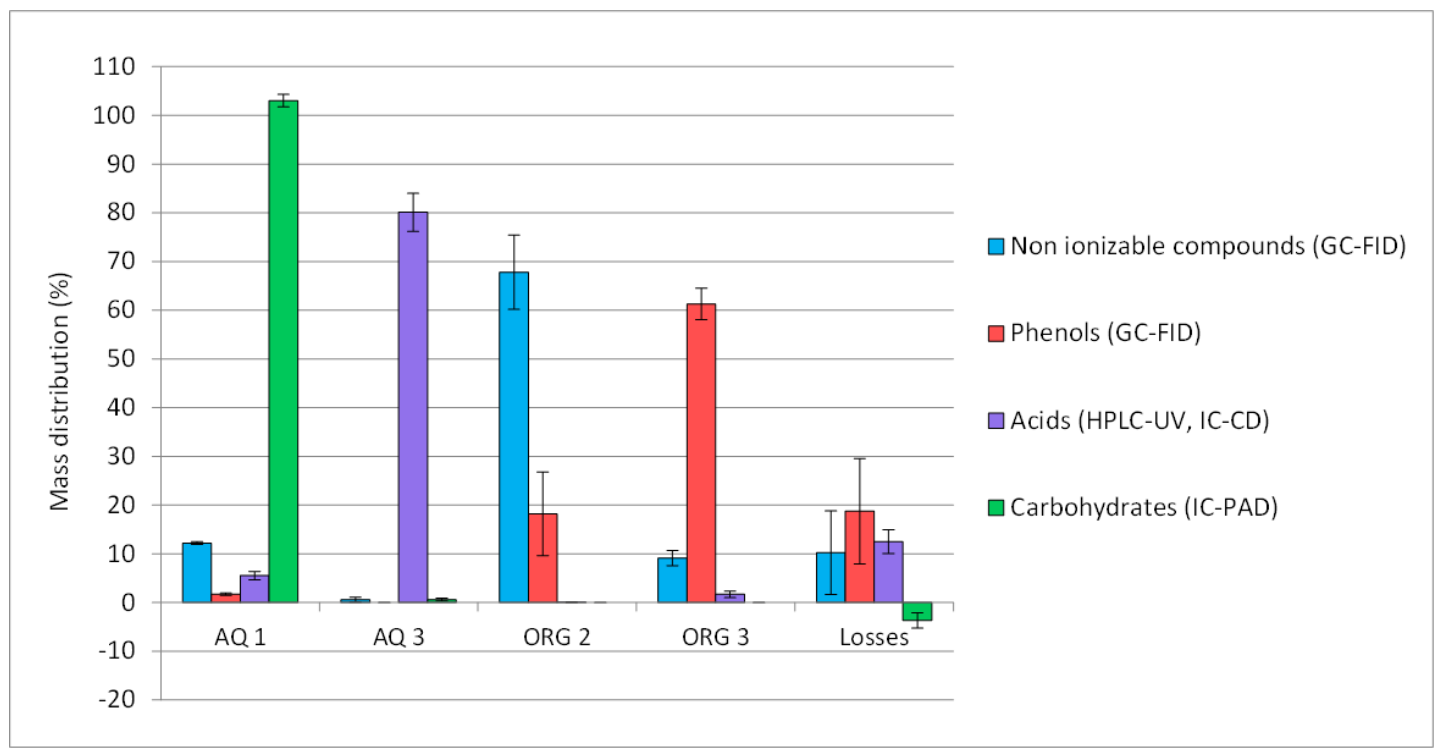


Figure S3: Venn diagrams representing the number of detected compounds with each ionization modes and those detected by several modes. Only the formulae of the compounds were used and compared between ionization modes to build this diagram. Thus isomers with different retention times were not taking into account and were considered as only one compound. (traced on http://bioinformatics.psb.ugent.be/cgi-bin/liste/Venn/calculate venn.htpl)

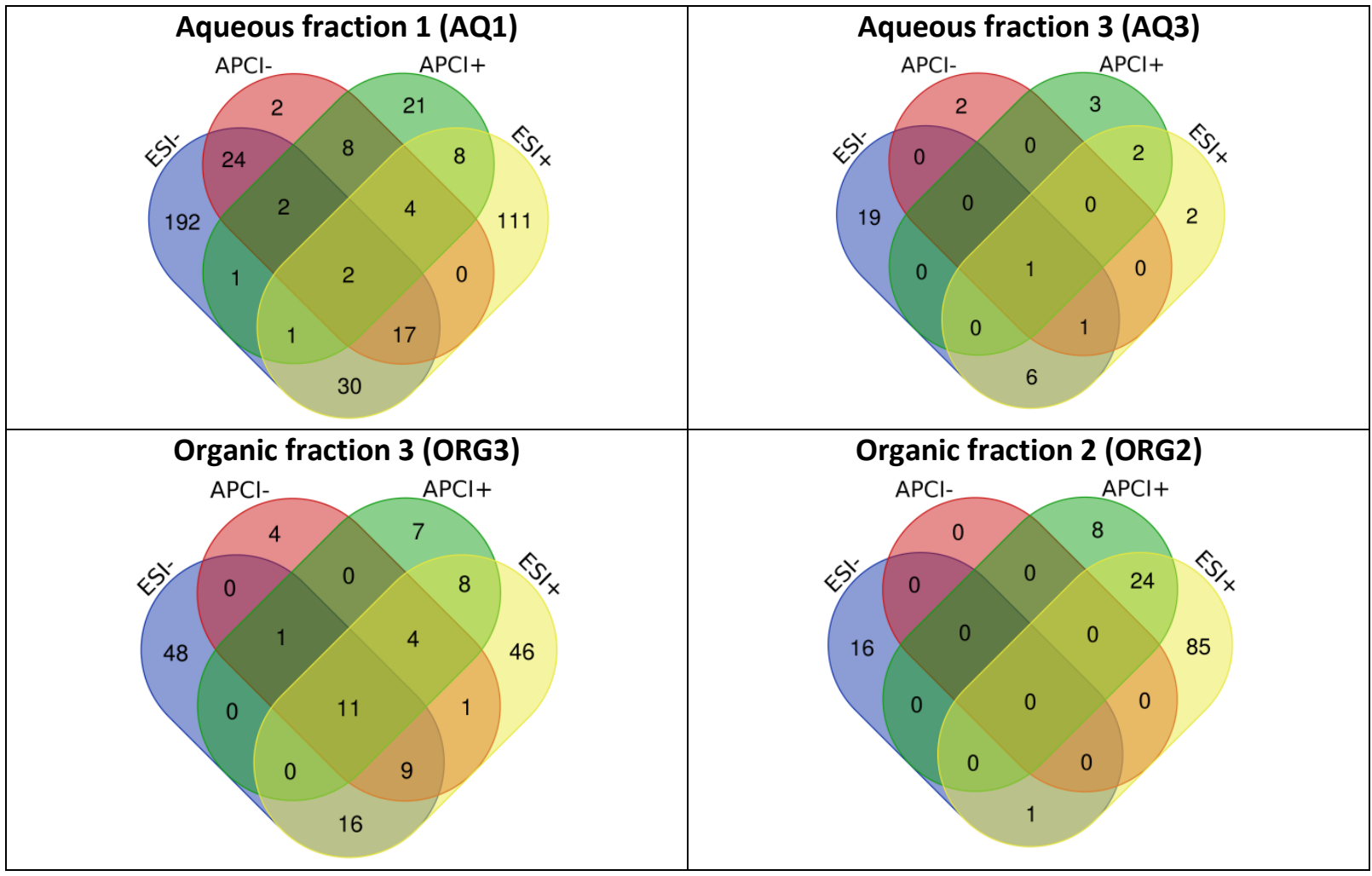

Figure S4: DBE of AQ1 components as a function of their molecular mass (blue points: compounds eluted with less than $12 \%$ of $\mathrm{MeOH}$, black points: eluted with more than $12 \%$ of $\mathrm{MeOH}$ )

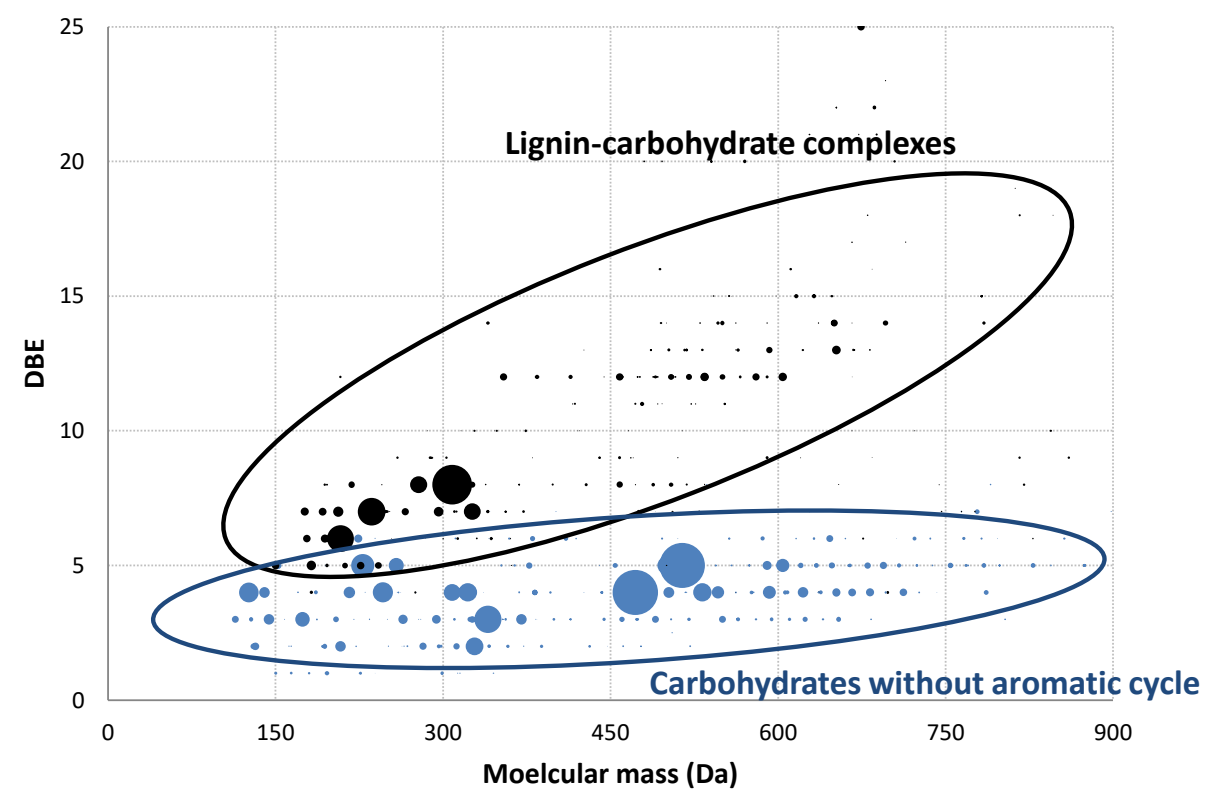


Figure S5: Main losses for $\mathrm{MS}^{2}$ to $\mathrm{MS}^{7}$ fragmentation stages in negative-ion mode ESI according to HPLC retention time (AQ1)

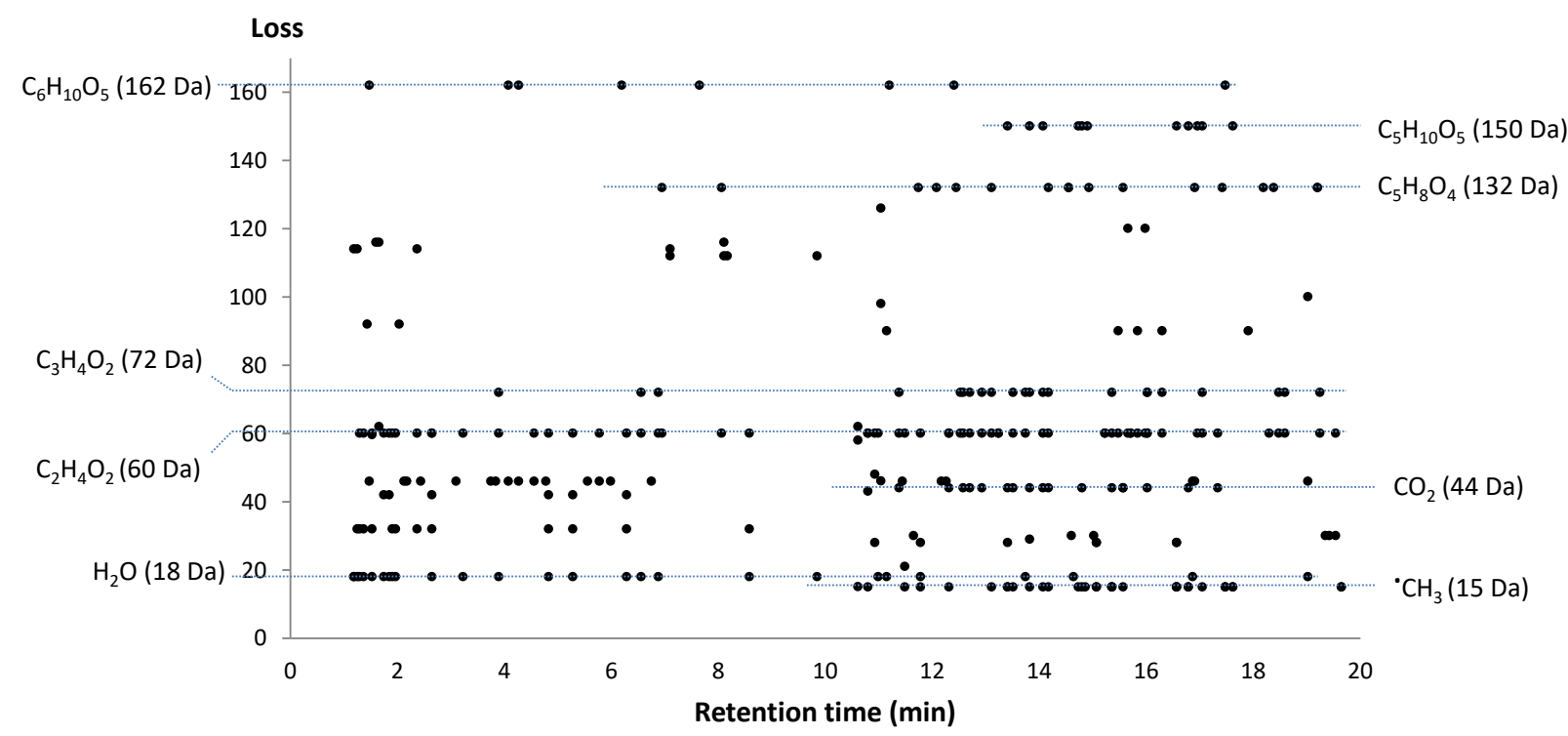

Figure S6: $\mathrm{MS}^{2}$ spectrum obtained in negative-ion ESI mode with annotation of compounds $\mathrm{a}, \mathrm{b}$ and $\mathrm{c}$ present in fraction $A Q 1$
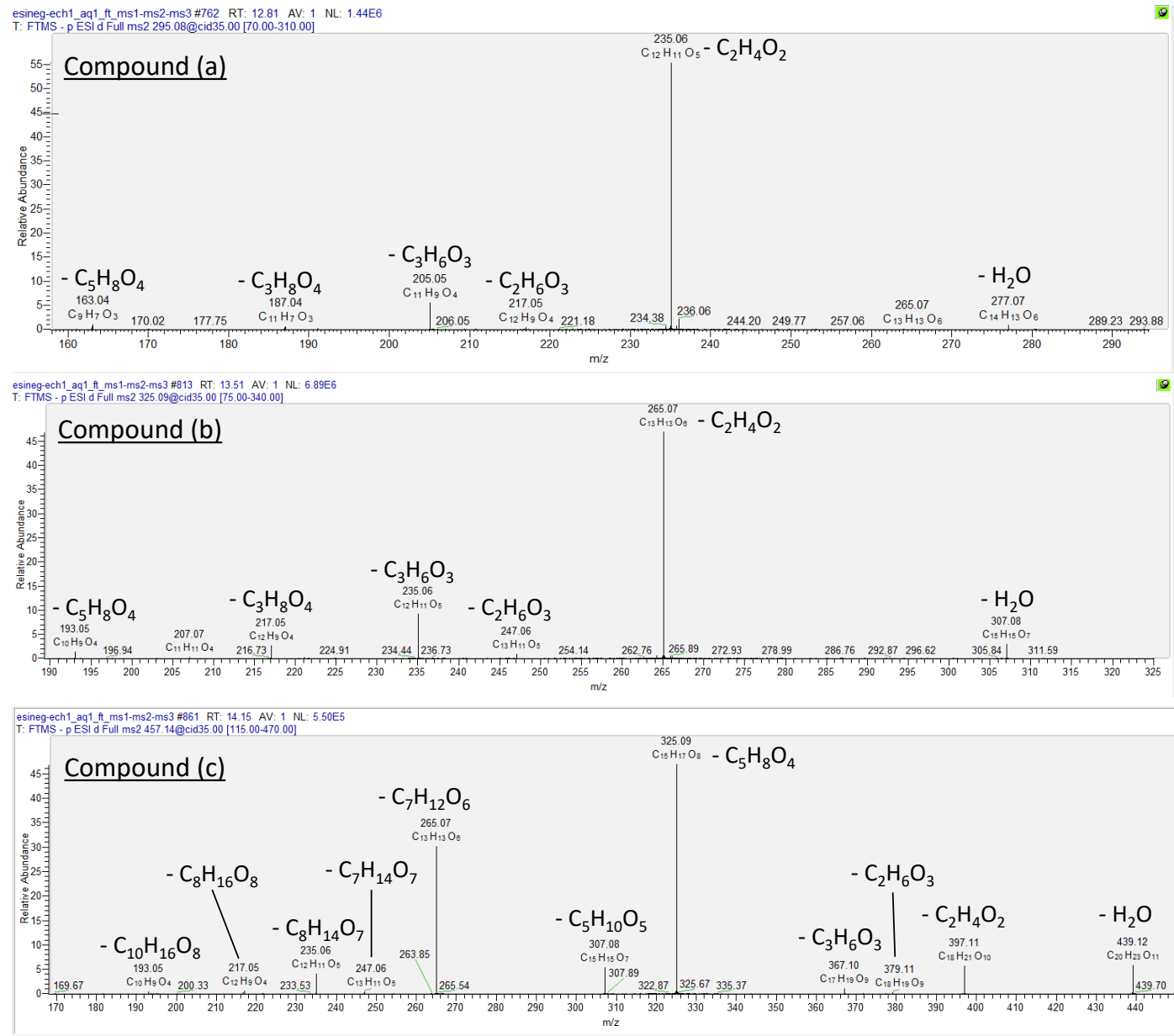
Figure S7: ORG2 van Krevelen diagram (1), DBE as a function of molecular mass (2) and neutral losses in positive-ion mode ESI for $\mathrm{MS}^{2}$ until MS3 according to retention time (3)
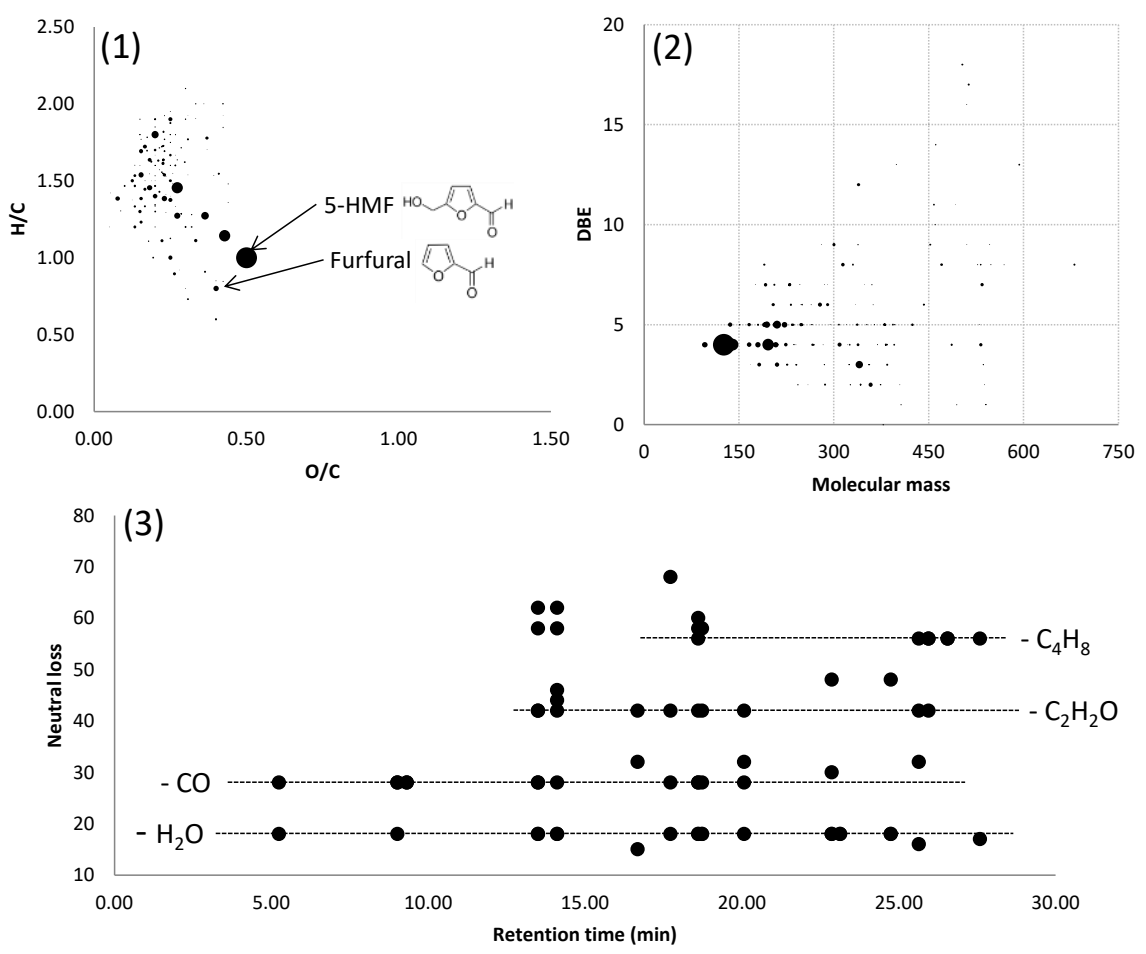

Figure S8: ORG3 van Krevelen diagram (1), DBE as a function of molecular mass (2) and losses in negative-ion mode ESI for $\mathrm{MS}^{2}$ (red dots) until MS6 (black outline circles) according to retention time (3)
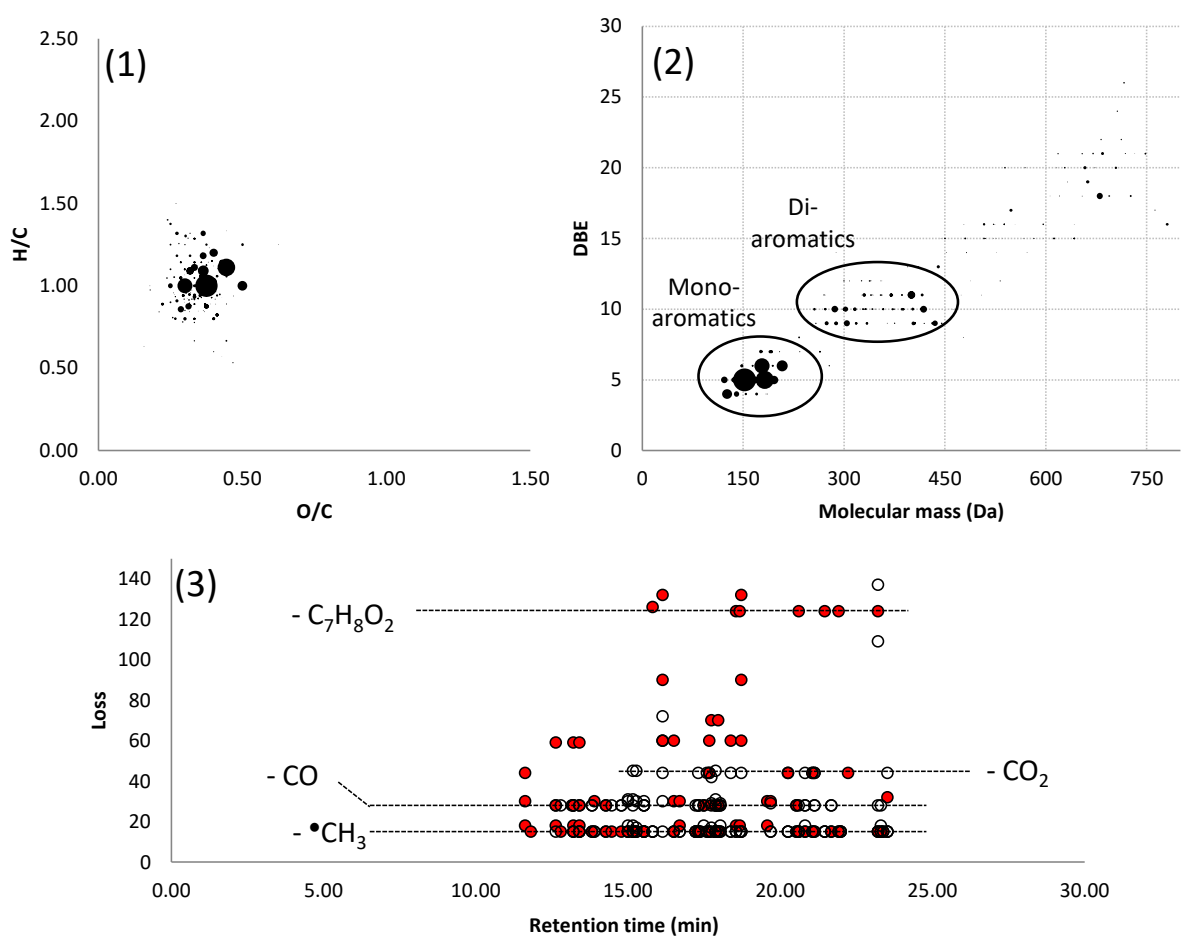
Table S2: Fragments obtained in negative-ion mode ESI for the most intense ions in AQ3

\begin{tabular}{|c|c|c|c|c|c|c|c|c|c|}
\hline$m / z$ & $t_{R}(\min )$ & $\begin{array}{l}\text { Ion formula } \\
(m / z)\end{array}$ & DBE & $\mathrm{MS}^{2}$ loss & $\mathrm{MS}^{3}$ loss & $\mathrm{MS}^{4}$ loss & $\mathrm{MS}^{5}$ loss & $\mathrm{Ms}^{6}$ loss & $\begin{array}{c}\text { Putative } \\
\text { identification }\end{array}$ \\
\hline 197.0456 & 12.1 & $\mathrm{C}_{9} \mathrm{H}_{9} \mathrm{O}_{5}$ & 5 & $\begin{array}{l}-\cdot \mathrm{CH}_{3}(100 \%) \\
-\mathrm{CO}_{2}(50 \%)\end{array}$ & $-\cdot \mathrm{CH}_{3}(100 \%)$ & & & & $\begin{array}{l}\text { Syringic acid } \\
\text { (DBE 5) }\end{array}$ \\
\hline 163.0400 & 13.2 & $\mathrm{C}_{9} \mathrm{H}_{7} \mathrm{O}_{3}$ & 6 & $-\mathrm{CO}_{2}(100 \%)$ & & & & & $\begin{array}{c}\text { Coumaric acid } \\
\text { (DBE 6) }\end{array}$ \\
\hline 193.0502 & 14.2 & $\mathrm{C}_{10} \mathrm{H}_{9} \mathrm{O}_{4}$ & 6 & $\begin{array}{l}-\cdot \mathrm{CH}_{3}(100 \%) \\
-\mathrm{CO}_{2}(75 \%) \\
-\left(\cdot \mathrm{CH}_{3}+\mathrm{CO}_{2}\right)(5 \%)\end{array}$ & $-\mathrm{CO}_{2}(100 \%)$ & & & & $\begin{array}{l}\text { Ferulic acid } \\
\text { (DBE 6) }\end{array}$ \\
\hline 187.0974 & 17.0 & $\mathrm{C}_{9} \mathrm{H}_{15} \mathrm{O}_{4}$ & 2 & $\begin{array}{l}-\mathrm{H}_{2} \mathrm{O}(100 \%) \\
-\left(\mathrm{H}_{2} \mathrm{O}+\mathrm{CO}_{2}\right)(30 \%)\end{array}$ & & & & & $\begin{array}{l}\text { Aliphatic acid } \\
\text { (DBE 2) }\end{array}$ \\
\hline 327.0873 & 13.0 & $\mathrm{C}_{18} \mathrm{H}_{15} \mathrm{O}_{6}$ & 11 & $\begin{array}{l}-\mathrm{CO}_{2}(100 \%) \\
-\left(2 \times \mathrm{CO}_{2}\right)(30 \%)\end{array}$ & $-\mathrm{CO}_{2}(100 \%)$ & & & & \\
\hline 389.1242 & 13.2 & $\mathrm{C}_{20} \mathrm{H}_{21} \mathrm{O}_{8}$ & 10 & $\begin{array}{l}-\mathrm{H}_{2} \mathrm{O}(100 \%) \\
-\left(\mathrm{CO}_{2}+\mathrm{H}_{2} \mathrm{O}\right)(10 \%) \\
-\mathrm{C}_{7} \mathrm{H}_{8} \mathrm{O}_{2}(5 \%) \\
-\mathrm{CO}_{2}(5 \%) \\
-\left(\mathrm{CO}_{2}+\mathrm{H}_{2} \mathrm{O}+\mathrm{CH}_{2} \mathrm{O}\right)(5 \%)\end{array}$ & $\begin{array}{l}-\mathrm{CO}_{2}(100 \%) \\
-\left(\mathrm{CO}_{2}+\mathrm{CH}_{2} \mathrm{O}\right)(5 \%)\end{array}$ & $\begin{array}{l}-\mathrm{CH}_{2} \mathrm{O}(100 \%) \\
-\mathrm{CH}_{3}(40 \%)\end{array}$ & $-\mathrm{CH}_{3}(100 \%)$ & $-\cdot \mathrm{CH}_{3}(100 \%)$ & \\
\hline 357.0978 & 13.5 & $\mathrm{C}_{19} \mathrm{H}_{17} \mathrm{O}_{7}$ & 11 & $\begin{array}{l}-\mathrm{CO}_{2}(100 \%) \\
-2 \times \mathrm{CO}_{2}(15 \%) \\
-\left(2 \times \mathrm{CO}_{2}+\mathrm{CH}_{3}\right)(10 \%)\end{array}$ & $\begin{array}{l}-\left(\mathrm{CO}_{2}+\mathrm{CH}_{3}\right)(100 \%) \\
-\mathrm{CO}_{2}(30 \%)\end{array}$ & & & & $\begin{array}{c}\text { Di-aromatic } \\
\text { compounds } \\
\text { (DBE between } \\
8 \text { and } 12 \text { ) }\end{array}$ \\
\hline 387.1083 & 13.8 & $\mathrm{C}_{20} \mathrm{H}_{19} \mathrm{O}_{8}$ & 11 & $\begin{array}{l}-\mathrm{CO}_{2}(100 \%) \\
-\left(2 \times \mathrm{CO}_{2}+\mathrm{CH}_{3}\right)(5 \%) \\
-\left(2 \times \mathrm{CO}_{2}\right)(5 \%)\end{array}$ & $\begin{array}{l}-\cdot \mathrm{CH}_{3}-\mathrm{CO}_{2}(100 \%) \\
-\mathrm{CO}_{2}(25 \%)\end{array}$ & $-\cdot \mathrm{CH}_{3}(100 \%)$ & & & \\
\hline 371.1133 & 14.4 & $\mathrm{C}_{20} \mathrm{H}_{19} \mathrm{O}_{7}$ & 11 & $\begin{array}{l}-\mathrm{H}_{2} \mathrm{O}(100 \%) \\
-\mathrm{CO}_{2}(40 \%) \\
-\mathrm{CH}_{2} \mathrm{O}(25 \%) \\
-\left(\mathrm{H}_{2} \mathrm{O}+\mathrm{CO}_{2}\right)(5 \%)\end{array}$ & $\begin{array}{l}-\mathrm{CO}_{2}(100 \%) \\
-\mathrm{CH}_{2} \mathrm{O}(30 \%) \\
-\left(\mathrm{CO}_{2}+\cdot \mathrm{CH}_{3}\right)(20 \%)\end{array}$ & $\cdot-\mathrm{CH}_{3}(100 \%)$ & $\begin{array}{l}-\mathrm{CH}_{3}(100 \%) \\
-\mathrm{CO}(20 \%)\end{array}$ & $\begin{array}{l}-\cdot \mathrm{CH}_{3}(100 \%) \\
-\mathrm{CO}(10 \%)\end{array}$ & \\
\hline
\end{tabular}




\begin{tabular}{|c|c|c|c|c|c|c|c|c|c|}
\hline 401.1240 & 17.7 & $\mathrm{C}_{21} \mathrm{H}_{21} \mathrm{O}_{8}$ & 11 & $\begin{array}{l}-\mathrm{H}_{2} \mathrm{O}(100 \%) \\
-\mathrm{CH}_{2} \mathrm{O}(30 \%) \\
-\mathrm{CO}_{2}(20 \%) \\
-\mathrm{C}_{10} \mathrm{H}_{10} \mathrm{O}_{5}(5 \%) \\
-\mathrm{C}_{9} \mathrm{H}_{10} \mathrm{O}_{3}(5 \%)\end{array}$ & $-\mathrm{CH}_{3}(100 \%)$ & $-\cdot \mathrm{CH}_{3}(100 \%)$ & & & \\
\hline 353.1030 & 17.8 & $\mathrm{C}_{20} \mathrm{H}_{17} \mathrm{O}_{6}$ & 12 & $-\mathrm{CH}_{3}(100 \%)$ & $-\cdot \mathrm{CH}_{3}(100 \%)$ & $-\mathrm{CO}_{2}(100 \%)$ & -CO (100\%) & & \\
\hline 537.1395 & 15.8 & $\mathrm{C}_{28} \mathrm{H}_{25} \mathrm{O}_{11}$ & 16 & $\begin{array}{l}-\mathrm{CO}_{2}(100 \%) \\
-2 \times \mathrm{CO}_{2}(30 \%) \\
-\mathrm{C}_{9} \mathrm{H}_{8} \mathrm{O}_{6}(5 \%)\end{array}$ & $-\mathrm{CO}_{2}(100 \%)$ & $-\cdot \mathrm{CH}_{3}(100 \%)$ & $\cdot \cdot \mathrm{CH}_{3}(100 \%)$ & $-\cdot \mathrm{CH}_{3}(100 \%)$ & \multirow{3}{*}{$\begin{array}{c}\text { Tri-aromatic } \\
\text { compounds } \\
\text { (DBE between } \\
12 \text { and } 18 \text { ) }\end{array}$} \\
\hline 477.1192 & 18.5 & $\mathrm{C}_{26} \mathrm{H}_{21} \mathrm{O}_{9}$ & 16 & $\begin{array}{l}-\mathrm{CO}_{2}(100 \%) \\
-\mathrm{C}_{2} \mathrm{H}_{4} \mathrm{O}_{3}(10 \%) \\
-\mathrm{C}_{8} \mathrm{H}_{8} \mathrm{O}_{4}(10 \%)\end{array}$ & $\begin{array}{l}-\cdot \mathrm{CH}_{3}(100 \%) \\
-\mathrm{CH}_{2} \mathrm{O}(10 \%)\end{array}$ & $-\cdot \mathrm{CH}_{3}(100 \%)$ & & & \\
\hline 523.1248 & 18.8 & $\mathrm{C}_{27} \mathrm{H}_{23} \mathrm{O}_{11}$ & 16 & $\begin{array}{l}-2 \times \mathrm{CO}_{2}(100 \%) \\
-\mathrm{CO}_{2}(95 \%)\end{array}$ & $\begin{array}{l}-\cdot \mathrm{CH}_{3}(100 \%) \\
-\mathrm{C}_{8} \mathrm{H}_{8} \mathrm{O}_{2}(60 \%)\end{array}$ & $-\cdot \mathrm{CH}_{3}(100 \%)$ & & & \\
\hline 731.1973 & 21.5 & $\mathrm{C}_{38} \mathrm{H}_{35} \mathrm{O}_{15}$ & 21 & $\begin{array}{l}-\mathrm{CO}_{2}(100 \%) \\
-\mathrm{H}_{2} \mathrm{O}-\mathrm{CO}_{2}(45 \%)\end{array}$ & & & & & \multirow{3}{*}{$\begin{array}{l}\text { Tretra- } \\
\text { aromatic } \\
\text { compounds } \\
\text { (DBE between } \\
18 \text { and } 24 \text { ) }\end{array}$} \\
\hline 699.2073 & 22.2 & $\mathrm{C}_{38} \mathrm{H}_{35} \mathrm{O}_{13}$ & 21 & $-\mathrm{CO}_{2}(100 \%)$ & & & & & \\
\hline 741.2182 & 22.4 & $\mathrm{C}_{40} \mathrm{H}_{37} \mathrm{O}_{14}$ & 22 & $\begin{array}{l}-\mathrm{CO}_{2}(100 \%) \\
-2 \times \mathrm{CO}_{2}(20 \%) \\
-\mathrm{C}_{20} \mathrm{H}_{20} \mathrm{O}_{7}(15 \%)\end{array}$ & $\begin{array}{l}-\mathrm{CO}_{2}(100 \%) \\
-\mathrm{C}_{19} \mathrm{H}_{20} \mathrm{O}_{5}(15 \%)\end{array}$ & & & & \\
\hline
\end{tabular}


Table S3: Fragments obtained in negative-ion mode ESI for the most intense ions in ORG3

\begin{tabular}{|c|c|c|c|c|c|c|c|c|c|}
\hline$m / z$ & $\operatorname{tr}(\mathrm{min})$ & $\begin{array}{l}\text { Ion formula } \\
(\mathrm{m} / \mathrm{z})\end{array}$ & DBE & $\mathrm{MS}^{2}$ loss & $\mathrm{Ms}^{3}$ loss & $\mathrm{Ms}^{4}$ loss & $\mathrm{Ms}^{5}$ loss & $\mathrm{Ms}^{6}$ loss & $\begin{array}{c}\text { Putative } \\
\text { identification }\end{array}$ \\
\hline 121.0293 & 10.1 & $\mathrm{C}_{7} \mathrm{H}_{5} \mathrm{O}_{2}$ & 5 & $-\mathrm{CO}(100 \%)$ & & & & & $\begin{array}{l}\text { Hydroxybenzaldehyde } \\
\text { (DBE 5) }\end{array}$ \\
\hline 151.0400 & 11.8 & $\mathrm{C}_{8} \mathrm{H}_{7} \mathrm{O}_{3}$ & 5 & $-\cdot \mathrm{CH}_{3}(100 \%)$ & $-\mathrm{CO}_{2}(100 \%)$ & & & & Vanillin (DBE 5) \\
\hline 181.0505 & 12.8 & $\mathrm{C}_{9} \mathrm{H}_{9} \mathrm{O}_{4}$ & 5 & $-\mathrm{CH}_{3}(100 \%)$ & $\cdot-\mathrm{CH}_{3}(100 \%)$ & $-\mathrm{CO}(100 \%)$ & & & $\begin{array}{l}\text { Syringaldehyde (DBE } \\
5 \text { ) }\end{array}$ \\
\hline 195.0661 & 13.8 & $\mathrm{C}_{10} \mathrm{H}_{11} \mathrm{O}_{4}$ & 5 & $-\mathrm{CH}_{3}(100 \%)$ & $\cdot \cdot \mathrm{CH}_{3}(100 \%)$ & $-\mathrm{CO}(100 \%)$ & $-\mathrm{CO}(100 \%)$ & & $\begin{array}{l}\text { Acetosyringone (DBE } \\
\text { 5) }\end{array}$ \\
\hline 177.0556 & 14.5 & $\mathrm{C}_{10} \mathrm{H}_{9} \mathrm{O}_{3}$ & 6 & $\begin{array}{l}-\cdot \mathrm{CH}_{3}(100 \%) \\
-\left(\cdot \mathrm{CH}_{3}+\mathrm{CO}\right)(60 \%)\end{array}$ & $-\mathrm{CO}(100 \%)$ & & & & $\begin{array}{l}\text { Coniferaldehyde (DBE } \\
6 \text { ) }\end{array}$ \\
\hline 207.0661 & 14.8 & $\mathrm{C}_{11} \mathrm{H}_{11} \mathrm{O}_{4}$ & 6 & $-\cdot \mathrm{CH}_{3}(100 \%)$ & $-\mathrm{CH}_{3}(100 \%)$ & $-\mathrm{CO}(100 \%)$ & $-\mathrm{CO}(100 \%)$ & & $\begin{array}{l}\text { Sinapaldehyde (DBE } \\
5 \text { ) }\end{array}$ \\
\hline 273.0765 & 12.6 & $\mathrm{C}_{15} \mathrm{H}_{13} \mathrm{O}_{5}$ & 9 & $\begin{array}{l}-\left(\mathrm{CO}+\mathrm{H}_{2} \mathrm{O}\right)(100 \%) \\
-\mathrm{H}_{2} \mathrm{O}(60 \%) \\
-\left(\cdot \mathrm{CH}_{3}+\mathrm{H}_{2} \mathrm{O}\right)(30 \%) \\
-\mathrm{CO}(25 \%) \\
-\mathrm{C}_{2} \mathrm{H}_{4} \mathrm{O}_{3}(20 \%) \\
-\mathrm{C}_{2} \mathrm{H}_{3} \mathrm{O}_{2}(20 \%)\end{array}$ & $\begin{array}{l}-\cdot \mathrm{CH}_{3}(100 \%) \\
-\mathrm{CO}(20 \%)\end{array}$ & & & & \\
\hline 303.0871 & 13.2 & $\mathrm{C}_{16} \mathrm{H}_{15} \mathrm{O}_{6}$ & 9 & $\begin{array}{l}-\left(\mathrm{CO}+\mathrm{H}_{2} \mathrm{O}\right)(100 \%) \\
-\mathrm{H}_{2} \mathrm{O}(95 \%) \\
-\left(\cdot \mathrm{CH}_{3}+\mathrm{H}_{2} \mathrm{O}\right)(60 \%) \\
-\cdot \mathrm{CH}_{3}(50 \%) \\
-\mathrm{CO}(30 \%) \\
-\mathrm{C}_{2} \mathrm{H}_{4} \mathrm{O}_{3}(10 \%) \\
-\mathrm{C}_{2} \mathrm{H}_{3} \mathrm{O}_{2}(5 \%)\end{array}$ & $\cdot \cdot \mathrm{CH}_{3}(100 \%)$ & $\cdot \cdot \mathrm{CH}_{3}(100 \%)$ & $-\mathrm{CO}(20 \%)$ & & $\begin{array}{c}\text { Di-aromatic } \\
\text { compounds (DBE } \\
\text { between } 8 \text { and 12) }\end{array}$ \\
\hline 333.0975 & 13.4 & $\mathrm{C}_{17} \mathrm{H}_{17} \mathrm{O}_{7}$ & 9 & $\begin{array}{l}-\left(\mathrm{CO}+\mathrm{H}_{2} \mathrm{O}\right)(100 \%) \\
-\mathrm{H}_{2} \mathrm{O}(95 \%) \\
-\cdot \mathrm{CH}_{3}(65 \%)\end{array}$ & $-\mathrm{CH}_{3}(100 \%)$ & $-\mathrm{CH}_{3}(100 \%)$ & $-\mathrm{CH}_{3}(100 \%)$ & $-\mathrm{CO}(20 \%)$ & \\
\hline
\end{tabular}




\begin{tabular}{|c|c|c|c|c|c|c|c|c|c|}
\hline & & & & $\begin{array}{l}-\mathrm{CO}(20 \%) \\
-\left(\cdot \mathrm{CH}_{3}+\mathrm{H}_{2} \mathrm{O}\right)(20 \%) \\
-\mathrm{C}_{2} \mathrm{H}_{3} \mathrm{O}_{2}(10 \%)\end{array}$ & & & & & \\
\hline 273.0765 & 17.3 & $\mathrm{C}_{15} \mathrm{H}_{13} \mathrm{O}_{5}$ & 9 & $-\mathrm{CH}_{3}(100 \%)$ & $\cdot \cdot \mathrm{CH}_{3}(100 \%)$ & $-\mathrm{CO}(100 \%)$ & -CO (100\%) & & \\
\hline 271.061 & 17.5 & $\mathrm{C}_{15} \mathrm{H}_{11} \mathrm{O}_{5}$ & 10 & $\begin{array}{l}-\left(\mathrm{CO}+\mathrm{CO}_{2}\right)(100 \%) \\
-\mathrm{CO}(40 \%)\end{array}$ & $\begin{array}{l}-\mathrm{H}_{2} \mathrm{O}(100 \%) \\
-\mathrm{CO}_{2}(50 \%)\end{array}$ & & & & \\
\hline 301.0716 & 17.7 & $\mathrm{C}_{16} \mathrm{H}_{13} \mathrm{O}_{6}$ & 10 & $\begin{array}{l}-\left(\mathrm{CO}+\mathrm{CO}_{2}+\mathrm{CH}_{3}\right)(100 \%) \\
-\cdot \mathrm{CH}_{3}(80 \%) \\
-\left(\mathrm{CO}+\mathrm{C}_{2} \mathrm{H}_{2} \mathrm{O}\right)(45 \%) \\
-\left(\mathrm{CO}+\mathrm{CO}_{2}\right)(30 \%) \\
-\mathrm{CO}(20 \%)\end{array}$ & $\begin{array}{l}-\mathrm{CHO}(100 \%) \\
-\mathrm{C}_{2} \mathrm{H}_{2} \mathrm{O}(30 \%) \\
-\mathrm{HO}(20 \%)\end{array}$ & & & & \\
\hline 299.0923 & 20.8 & $\mathrm{C}_{17} \mathrm{H}_{15} \mathrm{O}_{5}$ & 10 & $-\cdot \mathrm{CH}_{3}(100 \%)$ & $\cdot \cdot \mathrm{CH}_{3}(100 \%)$ & $\begin{array}{l}-\mathrm{CO}(100 \%) \\
-\mathrm{H}_{2} \mathrm{O}(15 \%)\end{array}$ & $\begin{array}{l}-\mathrm{CO}(100 \%) \\
-\mathrm{CO}_{2}(25 \%)\end{array}$ & & \\
\hline 329.0664 & 21.1 & $\mathrm{C}_{17} \mathrm{H}_{13} \mathrm{O}_{7}$ & 11 & $-\mathrm{CH}_{3}(100 \%)$ & $\cdot \cdot \mathrm{CH}_{3}(100 \%)$ & $\begin{array}{l}-\mathrm{CO}(100 \%) \\
-\left(\mathrm{CO}+\mathrm{CO}_{2}\right) \\
(25 \%)\end{array}$ & $-\mathrm{CO}_{2}(100 \%)$ & $\begin{array}{l}-\mathrm{CO}_{2}(100 \%) \\
-\mathrm{CO}(70 \%)\end{array}$ & \\
\hline 509.1454 & 18.6 & $\mathrm{C}_{27} \mathrm{H}_{25} \mathrm{O}_{10}$ & 15 & $\begin{array}{l}-\mathrm{H}_{2} \mathrm{O}(100 \%) \\
-\mathrm{C}_{7} \mathrm{H}_{8} \mathrm{O}_{2}(40 \%) \\
-\left(\mathrm{C}_{7} \mathrm{H}_{8} \mathrm{O}_{2}+\mathrm{CH}_{2} \mathrm{O}\right)(20 \%)\end{array}$ & $-\cdot \mathrm{CH}_{3}(100 \%)$ & $-\mathrm{CH}_{3}(100 \%)$ & & & \\
\hline 507.1299 & 21.9 & $\mathrm{C}_{27} \mathrm{H}_{23} \mathrm{O}_{10}$ & 16 & $\begin{array}{l}-\mathrm{CH}_{3}(100 \%) \\
-\mathrm{C}_{7} \mathrm{H}_{8} \mathrm{O}_{2}(85 \%) \\
-\left(2 \times \cdot \mathrm{CH}_{3}+\mathrm{CO}\right)(45 \%) \\
-\left(\cdot \mathrm{CH}_{3}+\mathrm{C}_{7} \mathrm{H}_{8} \mathrm{O}_{2}\right)(40 \%) \\
-\mathrm{C}_{10} \mathrm{H}_{10} \mathrm{O}_{3}(20 \%)\end{array}$ & $\begin{array}{l}-\left(\mathrm{CO}+\mathrm{CH}_{3}\right)(100 \%) \\
-\cdot \mathrm{CH}_{3}(80 \%)\end{array}$ & & & & $\begin{array}{c}\text { Tri-aromatic } \\
\text { compounds (DBE } \\
\text { between } 12 \text { and } 18 \text { ) }\end{array}$ \\
\hline 657.1983 & 21.4 & $\mathrm{C}_{36} \mathrm{H}_{33} \mathrm{O}_{12}$ & 20 & $\begin{array}{l}-\mathrm{C}_{18} \mathrm{H}_{16} \mathrm{O}_{6}(100 \%) \\
-\mathrm{C}_{7} \mathrm{H}_{8} \mathrm{O}_{2}(35 \%)\end{array}$ & $-\cdot \mathrm{CH}_{3}(100 \%)$ & $-\cdot \mathrm{CH}_{3}(100 \%)$ & $\begin{array}{l}-\cdot \mathrm{CH}_{3}(100 \%) \\
-\left(\cdot \mathrm{CH}_{3}+\mathrm{CO}\right) \\
(30 \%)\end{array}$ & & $\begin{array}{l}\text { Tretra-aromatic } \\
\text { compounds (DBE }\end{array}$ \\
\hline 683.2144 & 22.0 & $\mathrm{C}_{38} \mathrm{H}_{35} \mathrm{O}_{12}$ & 21 & $\begin{array}{l}-\mathrm{C}_{18} \mathrm{H}_{16} \mathrm{O}_{6}(100 \%) \\
-\mathrm{C}_{20} \mathrm{H}_{18} \mathrm{O}_{6}(90 \%)\end{array}$ & $-\cdot \mathrm{CH}_{3}(100 \%)$ & $-\cdot \mathrm{CH}_{3}(100 \%)$ & $-\cdot \mathrm{CH}_{3}(100 \%)$ & & between 18 and 24) \\
\hline
\end{tabular}

